\title{
Changes in reaction time, coefficient of variance of reaction time, and autonomic nerve function in the mental fatigue state caused by long-term computerized Kraepelin test workload in healthy volunteers
}

\author{
Daisuke Kuratsune $^{1}$, Seiki Tajima ${ }^{2}$, Junichi Koizumi ${ }^{3}$, Kouzi Yamaguti ${ }^{4}$, Tetsuya Sasabe ${ }^{4}$, \\ Kei Mizuno $^{5,6}$, Masaaki Tanaka ${ }^{6}$, Naoko Okawa ${ }^{7}$, Hideki Mito ${ }^{7}$, Hirokazu Tsubone ${ }^{8}$, \\ Yasuyoshi Watanabe ${ }^{5,6}$, Masayasu Inoue ${ }^{1}$, Hirohiko Kuratsune $e^{4,6,7,8}$ \\ ${ }^{1}$ Department of Biochemistry and Molecular Pathology, Graduate School of Medicine, Osaka City University, Osaka, Japan \\ ${ }^{2}$ Hyogo Children’s Sleep and Development Medical Research Center, Kobe, Japan \\ ${ }^{3}$ Division of Materials Science and Chemical Engineering, Yokohama National University, Yokohama, Japan \\ ${ }^{4}$ Clinical Center for Fatigue Science, Graduate School of Medicine, Osaka City University, Osaka, Japan \\ ${ }^{5}$ RIKEN, Center for Molecular Imaging Science, MI R\&D Center, Kobe, Japan \\ ${ }^{6}$ Department of Physiology, Graduate School of Medicine, Osaka City University, Osaka, Japan \\ ${ }^{7}$ Faculty of Health Science for Welfare, Kansai University of Welfare Sciences, Kasiwara, Japan \\ ${ }^{8}$ Department of Comparative Pathophysiology, Veterinary Medical Sciences, Graduate School of Agricultural \& Life Science, The \\ University of Tokyo, Tokyo, Japan \\ Email: kura@fuksi-kagk-u.ac.jp
}

Received 25 November 2011; revised 17 January 2012; accepted 16 February 2012

\begin{abstract}
Fatigue is a common sense caused by crushing labor, stressful social events and various illnesses. It is usually judged by their subjective symptoms, but it should be evaluated in an objective perspective. Here we show that the decrease of working efficiency and sympathetic hyperactivity are associated with mental fatigue state caused by prolonged mental workload. Recently we made a new mental fatigue model of healthy volunteers caused by long-term computerized Kraepelin test (CKT) workload. CKT is our new software for automatically checking the calculation capability, with which it is easy to determine the reaction time (RT), coefficient of variance of reaction time $(\mathrm{CV})$, and accuracy of the answers (AC) during tasks. We put 24 healthy volunteers into the fatigue state by subjecting them to 120 minutes' CKT workload, and then studied the changes in fatigue sensation, RT, CV, and AC before and after the CKT workload. The fatigue sensation, RT, and CV were clearly increased by the fatigue-inducing task and recovered during the resting period. We also studied the changes in autonomic nerve activity by using heart rate variability analysis. The low/high frequency component ratio (LF/HF) was significantly increased by the fatigue-inducing task and decreased by resting, suggesting that mental stress causes a relatively sympathetic nerve activity-dominant state. Therefore, our new fatigue model involve-
\end{abstract}

ing a long-term CKT workload is a good mental fatigue model to provide much information about the fatigue state simultaneously, and the increase of RT, $\mathrm{CV}$, and proportion of sympathetic activity (LF/HF) are associated with mental fatigue state. These might be useful objective biomarkers or evaluating a mental fatigue state.

Keywords: Fatigue; Computerized Kraepelin Test; Reaction Time; Accuracy of the Answers; Autonomic Nerve Activity

\section{INTRODUCTION}

It is well known that stressful social events frequently become the trigger for acute mental fatigue and sometimes cause problems with mental health and chronic fatigue, occasionally resulting in death in the case of overwork [1-3]. In Japan, $60 \%$ of the general adult population complaints of fatigue and one third of the population suffers from chronic fatigue [4]. Furthermore, about four hundred people have died caused by overwork every year. In 2006, the Japanese Ministry of Health, Labor, and Welfare made a new law for protecting against health problems due to overwork. According to this new law, Industrial physicians have to check the mental and physical health conditions of all laborers, if they work more than 100 hours overtime per month. However, no specific 
biomarkers for fatigue state have been found, and the vast majority of industrial physicians have judged their health conditions just by the subjective symptoms given in questionnaires and physiological observations. Therefore, Japanese physicians are extremely eager for evaluating worker's fatigue state objectively by using some makers of fatigue.

In 2009, the new study group for fatigue supported by the Japanese Ministry of Health, Labor, and Welfare (Head: Kuratsune $\mathrm{H}$ ) was set up, and the sensitivity and the specificity of several makers of fatigue state including autonomic nerve activity have been studied. Recently we demonstrated that autonomic nervous alterations are associated with daily level of fatigue [5], and that mental fatigue caused by prolonged cognitive load is associated with sympathetic hyperactivity [6].

Our co-workers K. Yamaguti and T. Sasabe devised the new software named the Computerized Kraepelin Test (CKT) for conveniently checking the calculation capability. Using this software, it is easy to determine the reaction time (RT), coefficient of variance of reaction time (CV), and accuracy of the answers (AC) during this test. Since the long-term CKT workload causes mental fatigue state, there is a possibility that a mental fatigue model of healthy volunteers would be produced by using this workload. Furthermore, as mentioned above, the CKT itself is the software for checking certain kinds of brain functions. Therefore, it also is available for checking changes in brain function during the fatigue state caused by the long-term CKT workload. In this present study we evaluated the fatigue sensation, and brain function in terms of $\mathrm{RT}, \mathrm{CV}, \mathrm{AC}$, and autonomic nerve function during, before and after the long-term CKT workload in healthy volunteers.

\section{MATERIALS AND METHODS}

\subsection{Subjects}

The subjects in this study were 24 students in Kansai University of Welfare Sciences (4 males and 20 females, 19 - 22 years of age). The study protocol complied with the Helsinki Declaration for Human Experimentation. The protocol was approved by the Ethics Committee of Kansai University of Welfare Science, and all subjects gave their written informed consent for the study.

\subsection{Protocol of Task}

We conducted a 2-day experiment. At the first and second morning without eating breakfast, all subjects assessed their subjective fatigue levels by using the visual analog scale (VAS) from 0 (minimum) to 100 (maximum). Their baseline autonomic nerve activities were determined by using heart rate variability analysis of the data from a 5-minute electrocardiogram (ECG) before the fatigue-inducing task. After this analysis, each subject performed a 2-hour fatigue-inducing task (see details in the following paragraph) without rest. After this task, they assessed their subjective fatigue levels by using VAS at 0,20 , and 60 minutes after the start of their rest period [7]. Their autonomic nerve activities were also examined again by 5-minute ECG before and after 20-, and 35-minute rest (Figure 1(a)). During the 2-hour fatigue-inducing task and 55-minute rest period, all subjects maintained the fasting state with the exception that they drank water.

\subsection{Computerized Kraepelin Test (CKT)}

In this study, computerized Kaepelin test was used as a fatigue-inducing task. Two digits from 0 to 9 and one "+" symbol were displayed at the top of the laptop computer screen (Figure 1(b)). Ten digits, from 0 to 9, were also displayed at the half bottom of the screen. The examinee was ordered to add these 2 digits at the top of the screen and show the answer by selecting a 1-digit number from 0 to 9 displayed at the half bottom of the screen by using a pointing device. For example, in the case of " $6+5$ " on the laptop computer screen, the answer is 11 ; but the examinee should select the last 1-digit number of 1 . When the examinee selected a 1-digit number on the screen, 2 new digits from 0 to 9 immediately appeared at the top of the screen. The examinee had to continue this procedure for 120 minutes as a fatigue-inducing task. The reaction time (RT), coefficient of variance of reaction time (CV), and accuracy of all of the answers (AC) were recorded automatically.

Since the response of most of the examinees during the first 5 minutes of this fatigue-inducing task was slow because of their inexperience, we evaluated the RT, CV, and $\mathrm{AC}$ at 6 - 10 minutes, 61 - 65 minutes, and 116 - 120 minutes after the beginning of the task as the change in response due to the fatigue state.

Furthermore, we also evaluated the RT, CV, and AC for 4 minutes after a $1^{\text {st }} 20$ - and a $2^{\text {nd }} 35$-minute rest for evaluating the change in the response due to their recovery by resting (Figure 1(a)).

\subsection{Measurement of Heart Rate Variability}

ECG signals were collected on-line via 3 electrodes attached to both wrists. These ECG signals were collected at $1000 \mathrm{~Hz}$ and converted from analog to digital with a 12-bit resolution. R-wave peaks were detected by using an LRR-03 device (GMS Co., Tokyo, Japan). R-R wave variability was measured as an indicator of autonomic nerve activity. The system calculated instantaneously the $\mathrm{R}-\mathrm{R}$ intervals as the difference between successive $\mathrm{R}$-wave peaks. 


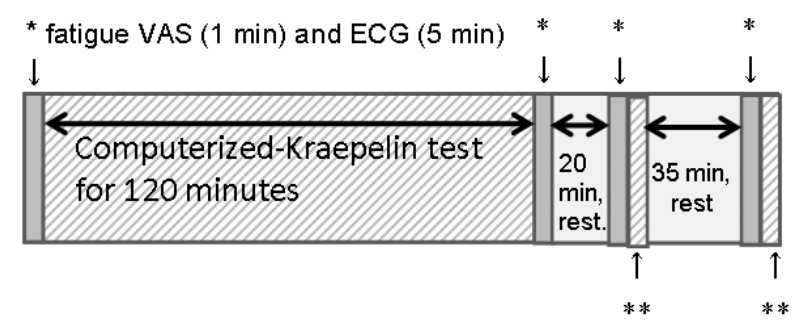

(a)

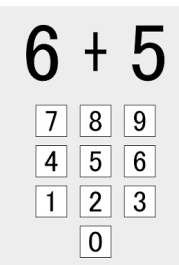

(b)

*: Subjects assessed their subjective fatigue levels for $1 \mathrm{~min}$ by using the visual analog scale (VAS), and their autonomic nerve activities were examined by heart rate variability analysis of the data from a 5-minute electrocardiogram (ECG); ${ }^{* *}$ : The RT, CV, and AC on the CKT lasting 4 minutes were also determined after 20 and 35 minutes' rest for evaluating their recovery by the rest.

Figure 1. (a) Schematic presentation of long-term Computerized Kraepelin Test (CKT) workload. We evaluated the reaction time (RT), coefficient of variance of reaction time (CV), and accuracy of the answers (AC) at 6 - 10 minutes, 61 - 65 minutes, and 116 - 120 minutes after the beginning of CKT as the change in response due to the fatigue state; (b) Schematic presentation of CKT on the laptop computer screen.

\subsection{Spectral Analysis}

Frequency-domain analyses of HRV were performed on a collected R-R interval time series of 5 consecutive minutes by using the maximum entropy method (MemCalc; GMS Co., Tokyo, Japan) [8]. Thirty-second subsequences of R-R intervals that started at every beat were generated. The power integrals of the low-frequency component (LF, 0.04 to $0.15 \mathrm{~Hz}$ ) and high-frequency component (HF, 0.15 to $0.40 \mathrm{~Hz}$ ), as well as the $\mathrm{LF} / \mathrm{HF}$ ratio, were calculated in all 30-second R-R interval subsequences. Finally, the mean of the LF/HF ratio in the whole time series was chosen as an autonomic index in this study.

\subsection{Statistical Analysis}

Differences in variables between groups were assessed by using a multivariate analysis of variance (type II MANOVA), which was performed on the fatigue VAS score, LF/HF ratio, RT, and CV of CKT. R version 2.8.0 was used to perform all multivariate analyses of variance [9].

\section{RESULTS}

\subsection{Behavioral Results}

The fatigue VAS (fVAS) scores of the 24 students before and after the long-term (2-hour) CKT workload are shown in Figure 2. The fVAS scores at both $1^{\text {st }}$ and $2^{\text {nd }}$ days were increased by the fatigue-inducing task and recovered to the baseline level by the rest. Those increases and recovery from the fatigue sensation were statistically signifycant (type II MANOVA, $\mathrm{p}<0.001$ ). The fVAS score for the morning of the $2^{\text {nd }}$ day ( $2^{\text {nd }}$ day baseline) tended to be higher than that for the morning of the $1^{\text {st }}$ day (type II MANOVA, $p=0.0547$ ).

Figure 3(a) shows the change in the RT of the 24 students before and after the CKT workload. Their RT was significantly increased by the fatigue-inducing task and recovered by the rest at both $1^{\text {st }}$ and $2^{\text {nd }}$ days (type II MANOVA, $\mathrm{p}<0.005)$. The RT of the $1^{\text {st }}$-day session during and after the CKT workload tended to be longer than that of the $2^{\text {nd }}$-day session (type II MANOVA, p = 0.093).

Figure 3(b) shows the change in the CV before and after the CKT workload. CV was also significantly increased by the fatigue-inducing task and recovered by the rest at both $1^{\text {st }}$ and $2^{\text {nd }}$ days (type II MANOVA, p < 0.001). For the $\mathrm{CV}$, there were no significant differences between the results of the $1^{\text {st }}$ and $2^{\text {nd }}$ days (type II MANOVA, $\mathrm{p}=0.549$ ).

The accuracy of task performance during and after the CKT workload was within the $98 \%$ to $100 \%$ range, and there was no significant difference in accuracy between during and after the fatigue-inducing task (data not shown).

\subsection{The Change of Autonomic Nerve Function}

Figure 4 shows the change in the LF/HF ratio of the fre-

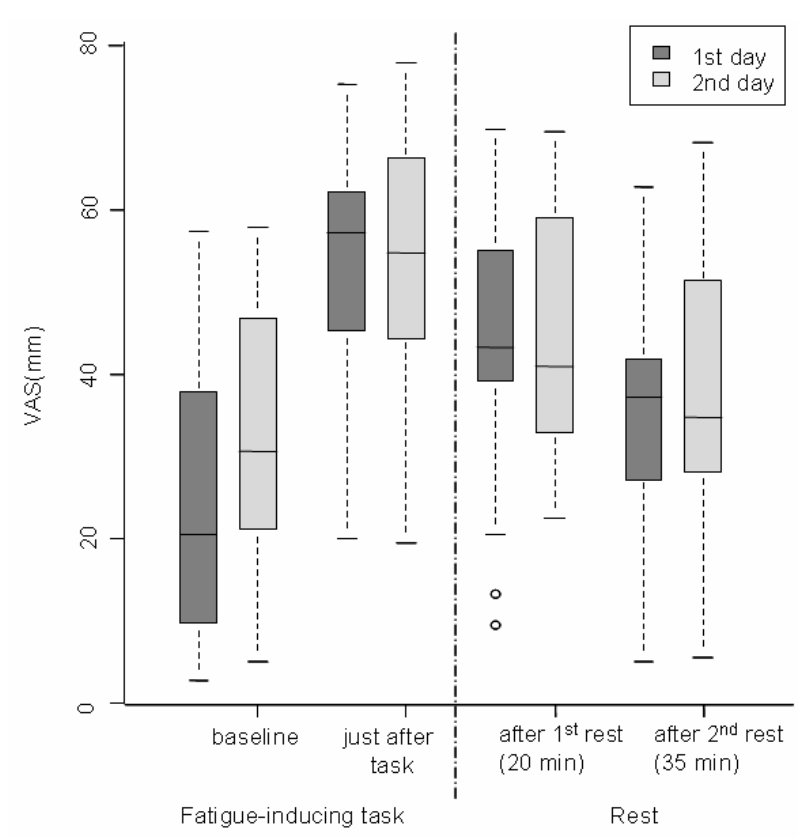

Figure 2. Subjective fatigue levels judged from visual analog scale (VAS) analysis before and after CKT workload. 


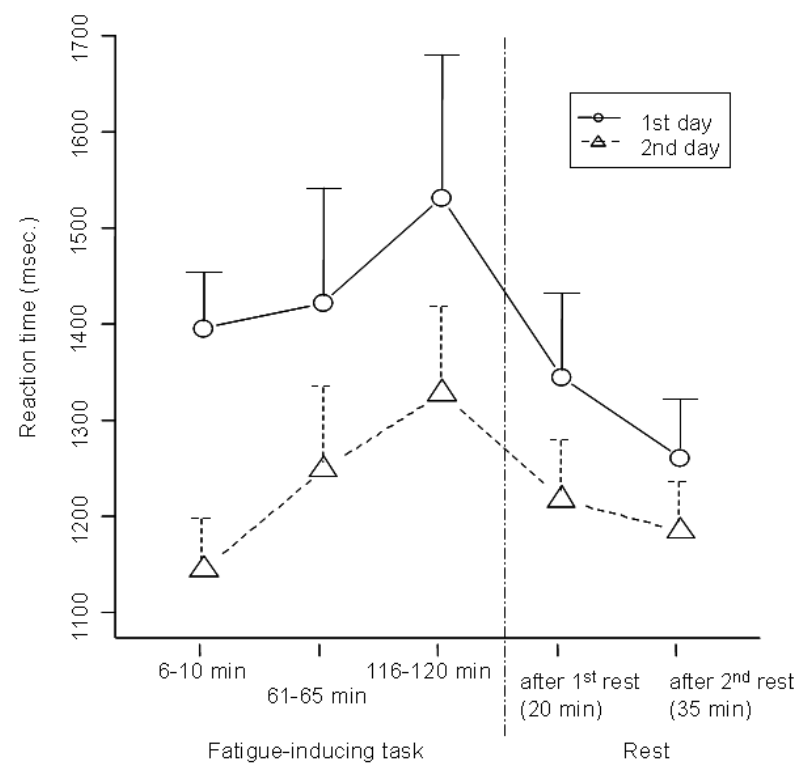

(a)

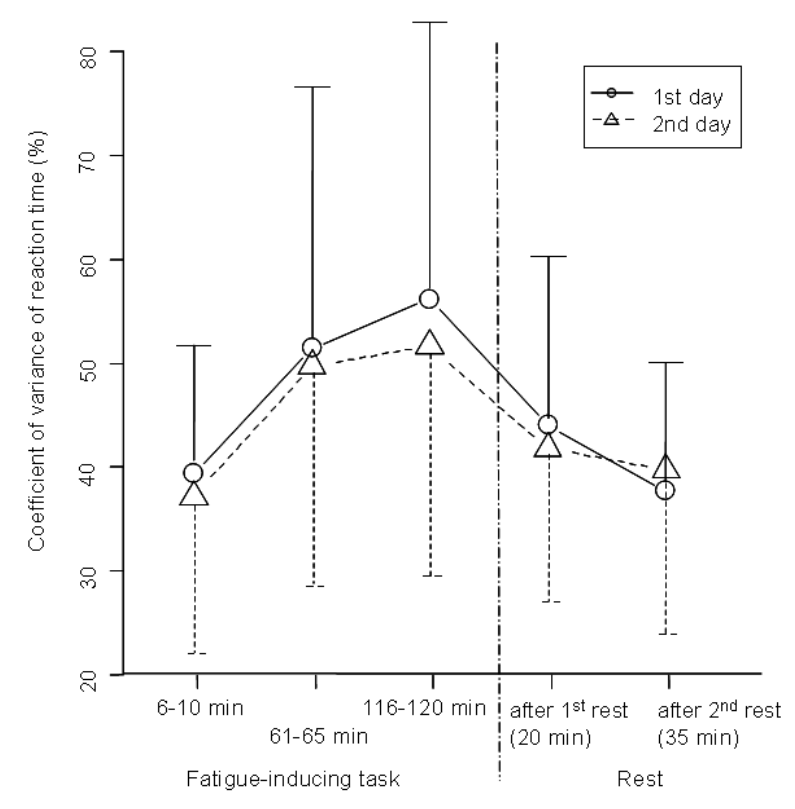

(b)

Figure 3. (a) Reaction Time (RT) before and after the CKT workload; (b) Coefficient of Variance (CV) of RT before and after the CKT workload.

quency domain spectrum obtained from the heart rate variability analysis. The LF/HF ratio was also significantly increased by the fatigue-inducing task and recovered by the rest at both $1^{\text {st }}$ and $2^{\text {nd }}$ days (type II MANOVA, $\mathrm{p}<0.05$ ). There were no significant differences between the results for the $1^{\text {st }}$ and $2^{\text {nd }}$ days (type II MANOVA, $\mathrm{p}$ $=0.411$ ).

\section{DISCUSSION}

The Uchida-Kraepelin psychodiagnostic (UKP) paper

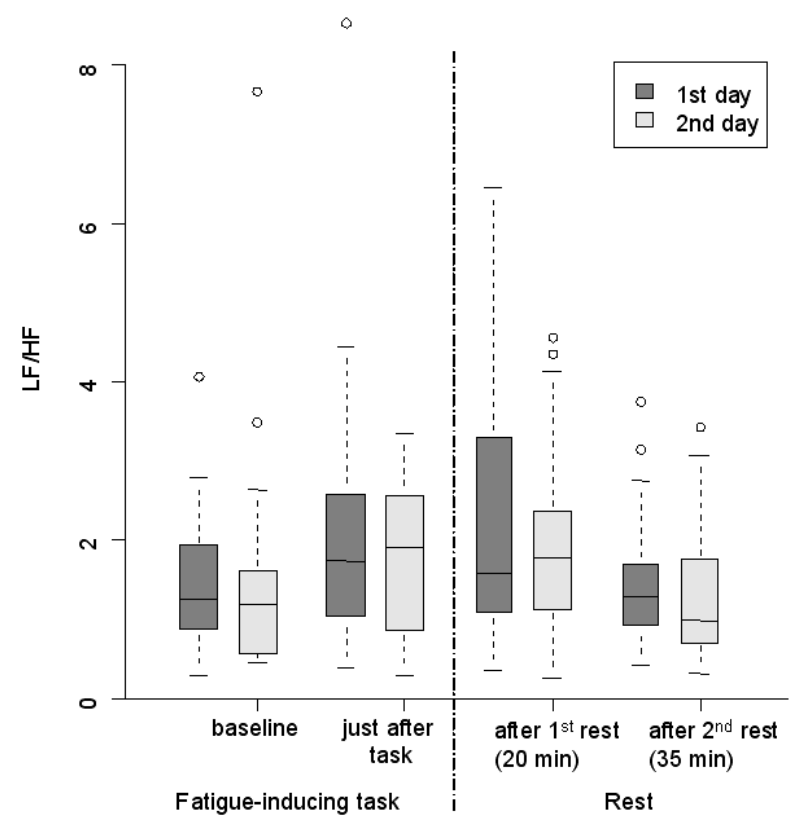

Figure 4. LF/HF ratio of frequency domain spectrum from heart rate variability analysis before and after the CKT workload.

test was proposed by Uchida Y in the 1920's. Successive pairs of digits arranged in long rows are added; and the integer in the unit column of each sum is noted. The examinee has to calculate and write the answers for 1 minute, and then directed the next row of digits. The examinee has to continue the nonstop calculations for 15 minutes, and after 5 minutes rest, the examinee must continue the calculations for 15 minutes again. Judging by the change in performance of calculation and accuracy, one is able to evaluate the characteristic features of character and behavior of the examinee. In Japan, this UKP paper test has been widely adopted as an aptitude test in Japan. However, the problem is that it requires much time and effort for checking the answers of the UKP paper test.

Recently, our co-workers K. Yamaguti and T. Sasabe devised the new software named the Computerized Kraepelin Test (CKT) for checking the above abilities automatically. Two digits from 0 to 9 are displayed on the laptop computer screen (Figure 1(b)), and the examinee has to add these 2 digits and show the answer by selecting 1 digit at the bottom of the display as soon as possible. By using this software, we could evaluate the characteristic features of character and behavior of the examinees automatically, and so we use this test not only for the original purpose of the UKP test, but also for evaluating certain kinds of brain function. When we evaluated the brain function in the fatigue state by using the CKT, we noticed that the long-term CKT itself caused mental fatigue [10]. 
As shown in Figures 2 and 3, 120 minutes CKT workload caused not only the increase of sense of fatigue, but also the prolongation of RT and CV during the workload. These changes were recovered by the resting period. Increase of RT is well known to be closely associated with fatigue state [11]. In our study, there is a significant correlation between RT and CV ( $r=0.528, \mathrm{p}<0.001)$, and incase of $\mathrm{CV}$ is also closely associated with the fatigue state caused by CKT workload.

When we used the traditional UKP paper test, it was possible to evaluate the number and the percentage of questions answered correctly per minute, but we could not evaluate the RT and the CV of each calculation. By using this CKT, it becomes to be clear that increase of $\mathrm{RT}$ and $\mathrm{CV}$ are closely associated with fatigue state.

The fVAS score at the morning of the $2^{\text {nd }}$ day tended to be higher than that for the morning of the $1^{\text {st }}$ day, indicating that the sense of fatigue caused by long-term CKT workload is not resolved by one-night's sleep. The RT of the $1^{\text {st }}$-day session during and after the CKT workload tended to be larger than that of the $2^{\text {nd }}$-day one. The reason for this difference might be related to the habituation effect.

The important point is that it is easily checking these changes of RT and CV in mental fatigue state by using our CKT workload. It is well known that various kinds of mental tasks cause the fatigue state, and lots of investigators have been studied the mechanisms of fatigue state using several fatigue models. However, there are a few models that make it possible to obtain the objective information about their fatigue state simultaneously. Therefore, by using this long-term CKT workload, a good model of mental fatigue was produced, because it is easy to obtain information about the RT, CV, and AC during the CKT.

As concerns the evaluation of autonomic nerve function by using power spectrum analysis of heart rate fluctuations, Akselrod et al. reported that sympathetic and parasympathetic nervous activities make frequency-specific contributions to the heart rate power spectrum [12]. Subsequent studies showed increased LF and reduced HF components of RR variability due to mental load or psychosocial stress $[13,14]$. Lecturing to the students reported to be resulted in a significant increase in both the LH/HF ratio and the secretion of the stress marker alpha-amylase [15]. The LH/HF ratio in power spectrum analysis of heart rate fluctuations is known to be associated with the proportion of sympathetic to parasympathetic nervous activity.

Recently, we revealed that not only the increased proportion of sympathetic activity (LF/HF ratio), but also the increased sympathetic activity and the decreased parasympathetic activity are associated with both the acute and daily level of fatigue [5]. We also found that these changes are associated with mental fatigue induced by prolonged cognitive load in healthy adults [6]. This study also confirms that the increased proportion of sympathetic activity (LF/HF ratio) is associated with mental fatigue state induced by another kind of mental task, 120 minutes CKT workload. When we studied the association between the behavioral results of CKT workload (RT, CT, AC) and autonomic nerve functions, we could not found any significant correlations among them, unfortunately. However, there is a tendency of weak negative correlation between CV and parasympathetic nerve activity $(r=-0.142$, $\mathrm{p}=0.059$ ) suggesting the decreased parasympathetic activity might be associated with the decrease of working efficiency. It seems to be a difficult to confirm the relationship between the fatigue-related phenomena and the change of autonomic nerve functions in a small study (n = 24). However, we believe that these fatigue-related phenomena might be associated with the deterioration of brain functions including autonomic nerve system, and it will be possible to demonstrate these associations, when we make an extensive study.

Indeed, when we studied the brain function of patients with severe fatigue state, chronic fatigue syndrome (CFS) by using ${ }^{15} \mathrm{O}$-labeled water $\left(\mathrm{H}_{2}{ }^{15} \mathrm{O}\right)$ and positron-emission tomography, we found that the vast majority of patients with CFS showed decreased blood flow in the anterior cingulated cortex, which is well known to be related to the autonomic nerve functions [16].

More recently, we also confirmed that the medial orbitofrontal cortex and a part of anterior cingulate cortex are associated with mental fatigue sensation, when we studied the change of brain function in the mental fatigue state induced by cognitive load in healthy adults [17]. Therefore, there is a close relationship between autonomic nerve function and fatigue state, and the results of autonomic nerve functions judged from the results of heart rate variability analysis seem to reflect the brain dysfunction in the fatigue state. We are now studying the direct association between the autonomic nerve functions by using heart rate variability analysis and brain function assessed by electroencephalography, functional magnetic resonance imaging, and magnetoencephalography in the mental fatigue model.

\section{CONCLUSION}

The long-term CKT workload provides a good mental fatigue model, because with this model it is easy to obtain information about fatigue sensation, RT, CV, and AC simultaneously. The study of autonomic nerve activity by heart rate variability analysis can also be done for evaluating the fatigue state. From this study, it become clear that the increase of RT, CV, and proportion of sympathetic activity (LF/HF ratio) are associated with 
mental fatigue state, and these are useful objective biomarkers for evaluating a mental fatigue state.

\section{ACKNOWLEDGEMENTS}

The authors are indebted to Mr. Genki Kosuge and Junpei Maeda (Division of Materials Science and Chemical Engineering, Faculty of Engineering, Yokohama National University) for their generous help in the long-term CKT workload. This work was supported by grants from Japanese Ministry of Health, Labor and Welfare. This work was also supported in part by a special grant by Kansai University of Welfare Sciences.

\section{REFERENCES}

[1] Uchiyama, S. Kurasawa, T. Sekizawa T. and Nakatsuka H. (2005) Job strain and risk of cardiovascular events in treated hypertensive Japanese workers: Hypertension follow-up group study. Journal of Occupational Health, 47, 102-111. doi:10.1539/joh.47.102

[2] Mizuno-Lewis, S. and McAllister, M. (2008) Taking leave from work: The impact of culture on Japanese female nurses. Journal of Clinical Nursing, 17, 274-281.

[3] Iwasaki, K., Takahashi, M. and Nakata, A. (2006) Health problems due to long working hours in Japan: working hours, workers' compensation (Karoshi), and preventive measures. Industrial Health, 44, 537-540. doi:10.2486/indhealth.44.537

[4] Watanabe, Y. (2008) Preface and mini-review: Fatigue science for human health. In: Watanabe, Y., Evengard, B., Natelson, B.H., Jason, L.A. and Kuratsune, H., Eds., Fatigue Science for Human Health, Springer, New York, 5-11.

[5] Tanaka, M., Mizuno, K., Yamaguti, K., Kuratsune, H., Fujii, A., Baba, H., Matsuda, K., Nishimae, A., Takesaka, T. and Watanabe, Y. (2011) Autonomic nervous alterations associated with daily level of fatigue. Behavioral and Brain Functions, 7, 46. http://www.ncbi.nlm.nih.gov/pmc/articles/PMC3214128/ pdf/1744-9081-7-46.pdf

[6] Mizuno, K., Tanaka, M., Yamaguti, K., Kajimoto, O., Kuratsune, H. and Watanabe, Y. (2011) Mental fatigue caused by prolonged cognitive load associated with sympathetic hyperactivity. Behavioral and Brain Functions, 7, 17.

http://www.ncbi.nlm.nih.gov/pmc/articles/PMC3113724/ pdf/1744-9081-7-17.pdf

[7] Lee, K.A., Hicks, G. and Nino-Murcia, G. (1991) Validity and reliability of a scale to assess fatigue. Psychiatry Research, 36, 291-298. doi:10.1016/0165-1781(91)90027-M
[8] Shinba, T., Kariya, N., Matsui, Y., Ozawa, N., Matsuda, Y. and Yamamoto, K. (2008) Decrease in heart rate variability response to task is related to anxiety and depressiveness in normal subjects. Psychiatry and Clinical Neurosciences, 62, 603-609. doi:10.1111/j.1440-1819.2008.01855.x

[9] Ihaka, R. and Gentleman, R. (1996) A language for data analysis and graphics. Journal of Computational and Graphical Statistics, 5, 299-314.

[10] Ataka, S., Tanaka, M., Nozaki, S., Mizuma, H., Mizuno, K., Tahara, T., Sugino, T., Shirai, T., Kajimoto, Y., Kuratsune, H., Kajimoto, O. and Watanabe, Y. (2008) Effects of oral administration of caffeine and D-ribose on mental fatigue. Nutrition, 24, 233-238. doi:10.1016/j.nut.2007.12.002

[11] Stefank, K. (1923) Reaction time and fatigue. Science, 57, 204-205. doi:10.1126/science.57.1468.204-a

[12] Akselrod, S., Gordon, D., Ubel, F.A., Shannon, D.C., Berger, A.C. and Cohen, R.J. (1981) Power spectrum analysis of heart rate fluctuation: A quantitative probe of beat-to-beat cardiovascular control. Science, 213, 220222. doi:10.1126/science.6166045

[13] Pagani, M., Furlan, R., Pizzinelli, P., Crivellaro, W., Cerutti, S. and Malliani, A. (1989) Spectral analysis of $\mathrm{R}-\mathrm{R}$ and arterial pressure variabilities to assess sympatho-vagal interaction during mental stress in humans. Journal of Hypertension, 6S, 14-15.

[14] Lucini, D., Di Fede, G., Parati, G. and Pagani, M. (2005) Impact of chronic psychosocial stress on autonomic cardiovascular regulation in otherwise healthy subjects. $\mathrm{Hy}$ pertension, 46, 1201-1206. doi:10.1161/01.HYP.0000185147.32385.4b

[15] Filaire, E., Portier, H., Massart, A., Ramat, L. and Teixeira, A. (2010) Effect of lecturing to 200 students on heart rate variability and alpha-amylase activity. European Journal of Applied Physiology, 108, 1035-1043. doi:10.1007/s00421-009-1310-4

[16] Kuratsune, H., Yamaguti, K., Lindh, G., Evengård, B., Hagberg, G., Matsumura, K., Iwase, M., Onoe, H., Takahashi, M., Machii, T., Kanakura, Y., Kitani, T., Långström, B. and Watanabe, Y. (2002) Brain regions involved in fatigue sensation: Reduced acetylcarnitine uptake into the brain. Neuroimage, 17, 1256-1265. doi:10.1006/nimg.2002.1260

[17] Tajima, S., Yamamoto, S., Tanaka, M., Kataoka, Y., Iwase, M., Yoshikawa, E., Okada, H., Onoe, H., Tsukada, H., Kuratsune, H., Ouchi, Y. and Watanabe, Y. (2010) Medial orbitofrontal cortex is associated with fatigue sensation. Neurology Research International, 2010, Article ID: 671421. doi:10.1155/2010/671421 http://www.ncbi.nlm.nih.gov/pmc/articles/PMC3003967/ pdf/NRI2010-671421.pdf 